\title{
LA INSERCIÓN PROFESIONAL DE LOS LICENCIADOS EN PSICOPEDAGOGÍA DE LA UNIVERSIDAD DE CASTILLA-LA MANCHA: EXPLORACIÓN DEL PERFIL DEL ALUMNADO
}

\section{ENTRY INTO THE LABOUR MARKET OF PSYCHOPEDAGOGY GRADUATE STUDENTS OF THE UNIVERSITY OF CASTILLA-LA MANCHA: ANALYSIS OF THE STUDENT'S PROFILE}

\author{
Asunción Manzanares*, Sara Ulla** y María José Galván*** \\ Universidad de Castilla-La Mancha
}

\section{RESUMEN}

El presente trabajo forma parte de un proyecto de mayor envergadura cuyo objetivo principal es la identificación de los factores que pueden facilitar o dificultar la inserción profesional de los Psicopedagogos de la Universidad de Castilla-La Mancha. El trabajo que ahora presentamos tiene como objeto el estudio detallado de las características de la muestra que está siendo y será sujeto de inserción profesional. A la vista de los resultados cabe destacar la modificación general que se está produciendo en la región en lo referido a la fuerza de trabajo, elevándose claramente el grado de cualificación en la generación evaluada con respecto a la anterior. Por otra parte, factores como la carencia de estrategias de desarrollo de la carrera y el bajo conocimiento en estrategias específicas de búsqueda de empleo como psicopedagogos pueden dificultar la inserción. Sin embargo, es destacable la movilidad territorial del alumnado para estudiar, con lo que es esperable que la redistribución en el territorio funcione como factor de potenciación de la inserción profesional de este colectivo.

Palabras clave: Orientación profesional. Inserción profesional. Transición universidad-empleo.

* Doctora en Filosofía y Ciencias de la Educación por la Universidad Complutense de Madrid. Experiencia profesional vinculada al ámbito de la Pedagogía Laboral (formación continua y orientación profesional).

** Doctora en Psicología por la Universidad Autónoma de Madrid. Experiencia profesional vinculada a distintas áreas de la Evaluación Psicológica.

*** Licenciada en Filosofía y Ciencias de la Educación (Sección Psicología) por la Universidad de Valencia. Experiencia profesional vinculada a las áreas del Desarrollo y la Educación. 


\begin{abstract}
The present report is enclosed in a larger research project that points toward the identification of the factors that could modify transition from University to work in Psycho-pedagogy of the Universidad de Castilla-La Mancha. The main aim of this paper is to present a detailed analysis and description of the sample characteristics that will have to insert in the region. According to the results, an important change in the regional work force has occurred. An important increase in the cohort qualification has been observed, compared with the previous age cohort. Factors as the low strategies knowledge to search for an adequate employment can impair the professional insertion. On the other hand, a high regional mobility to go to University has been observed in the sample, so we can expect that graduate-students redistribution will ease professional insertion.
\end{abstract}

Key words: Vocational guidance. Career development. Transition university and work.

\title{
Introducción
}

El trabajo que aquí presentamos forma parte de un estudio de mayor envergadura y con carácter longitudinal, cuyo objetivo último es la identificación de los factores determinantes del proceso de inserción profesional de los Psicopedagogos de la Universidad de CastillaLa Mancha que iniciaron sus estudios en el curso académico 1998/99 en el Campus de Cuenca. El proyecto en curso se suma al conjunto de investigaciones que, ya con cierta tradición en nuestro país -sobre todo en las décadas de los ochenta y noventa y ahora con nuevas formulaciones y preocupaciones-, se han dedicado al análisis del proceso de inserción profesional en el ámbito diferencial de la universidad. No obstante, es su adscripción territorial la que dota de ciertas peculiaridades e importancia al estudio. En primer lugar, en el conjunto de la oferta de la Universidad de Castilla-La Mancha, los estudios de Psicopedagogía se imparten desde 1998, gozan de la condición de nuevas enseñanzas, lo que a efectos de alcance del estudio tiene dos implicaciones claras: a) en este momento (julio 2001), sólo hay dos promociones de licenciados en situación de búsqueda activa de empleo como psicopedagogos o ejerciendo como tales desde hace solo un año; b) ha transcurrido un tiempo mínimo para constatar la pertinencia social de estos estudios en el contexto de acción que le es propio, así como su capacidad de ofertar una formación comparable al resto del territorio y verse afectada por procesos similares de demanda de titulados.

En segundo lugar, cabe apuntar que en este año se ha culminado el proceso de transferencia de competencias educativas a la Comunidad, de forma que se abren nuevas posibilidades en la organización y gestión de la cuestión educativa. Con respecto a la satisfacción de las necesidades educativas de la región, el Libro Blanco de la Educación en Castilla-La Mancha (Consejería de Educación, 1999) ofrece un vasto panorama y posibilidades laborales para los profesionales de la educación, entre ellos para los psicopedagogos. No es nuestra intención describir aquí el exhaustivo análisis que de la situación de la educación en la región ofrece el Libro Blanco, pero sí creemos conveniente, para situar al lector, apuntar algunas características que definen el escenario de inserción y que matizan el perfil y las funciones del psicopedagogo en este contexto:

1. Castilla-La Mancha es una región extensa, poco poblada $\left(21,6 \mathrm{hab} / \mathrm{km}^{2}\right)$ y con una estructura de asentamiento poblacional marcadamente rural. Cuenta con una elevada dispersión geográfica de los centros educativos (un total de 1.070), planteándose 
como problemática la estabilidad del profesorado en los centros ubicados en zonas rurales.

2. Los servicios educativos de apoyo a los centros (CPR, EOEP y equipo de inspección) desarrollan su función en extensas zonas educativas. A este respecto, es importante señalar que el desarrollo de la orientación psicopedagógica desde los EOPs se encuentra con la dificultad que supone intervenir, como media, en seis centros educativos, con 1.200 alumnos.

3. La educación en esta región se presenta como un innegable factor de compensación de desigualdades en el acceso, promoción y permanencia en el sistema educativo, así como una importante herramienta para hacer frente a algunos problemas emergentes y potencialmente conflictivos como puede ser la inmigración e integración social en el sector agrícola, la diversidad étnica y cultural creciente y la atención a los alumnos con necesidades educativas especiales. Todos ellos son espacios privilegiados para la intervención psicopedagógica.

4. La ausencia de licenciados propios en Psicología y Pedagogía, define a priori un mercado de trabajo más abierto para los psicopedagogos castellano-manchegos que, en gran medida, explica la cualificación particular que están recibiendo. La licenciatura en Psicopedagogía en nuestra Universidad consta de dos itinerarios formativos diferenciados: uno, escolar, que especializa en ámbitos de intervención reglados y conforme al perfil habitual del psicopedagogo; otro, sociolaboral, que cualifica para la acción en contextos menos reglados y en los que sobresalen las implicaciones de la acción psicopedagógica de tipo social (desarrollo comunitario) y vinculada al mundo del trabajo (formación en organizaciones empresariales, promoción del empleo).

\section{Antecedentes y bases del estudio}

El análisis de los procesos de transición desde el sistema educativo al mercado laboral es un tema tradicional de investigación en el ámbito de las ciencias sociales y de la educación. Desde campos de conocimiento como la sociología, la economía, la pedagogía o la psicología, se han realizado estudios focalizados en poblaciones diversas que, desde diferentes planteamientos metodológicos y con distinto poder explicativo y capacidad predictiva, han contribuido a una progresiva definición de los factores determinantes de la integración a la vida activa.

Llegar a definir este proceso no es una tarea fácil. En primer lugar, porque la transición a la vida activa, en tanto que proceso, es móvil y dinámica y, en segundo término, porque los factores detectados que interactúan sobre la transición son cambiantes en función de la propia evolución de la economía y de los valores sociales vigentes en un determinado momento. Fenómenos como la reestructuración de los procesos productivos y su repercusión sobre la segmentación del mercado, la organización del trabajo o el juego entre la oferta y demanda de fuerza de trabajo, junto con la consiguiente proliferación de nuevas formas de actividad laboral, han transformado los procesos de integración a la vida activa rompiendo la visión uniforme y lineal, característica de tiempos pasados. Roquero (1995) señala a este respecto que «cuando se producen itinerarios desestructurados por fracaso escolar y/o profesional, cuando otros compaginan estudios y trabajo, cuando la contratación es discontinua 
y aparece la remuneración en ausencia de relación contractual, sólo cabe abordar la integración laboral desde una visión alejada de los perfiles simples y lineales sobre la situación de estudiante, demandante de empleo y trabajador» (pág.21).

Son muchas las razones que explican que esta línea de investigación haya sido profusamente alimentada, especialmente en los momentos en que el mercado laboral se caracteriza por una elevada tasa de paro y cuando éste afecta a poblaciones especialmente vulnerables como es el caso de los jóvenes menores de 30 años y en un contexto determinado como puede ser el europeo. En el caso concreto de los estudios realizados en el campo de la inserción profesional de los graduados, señala De Miguel (tomado de Spenner, 1985) que la mayoría de los resultados conseguidos están determinados por el enfoque adoptado en la definición de las relaciones entre educación superior y empleo. Nos atrevemos a afirmar que todos estos estudios consideran que la incorporación al trabajo es una de las transiciones más importantes de la vida adulta, sin embargo, difieren en el enfoque desde el que abordan esa relación. Así, encontramos análisis economicistas de los resultados académicos y del papel que los estudios universitarios desempeñan en el conjunto social (vinculados, por ejemplo, a la teoría del capital humano o a la del credencialismo) y, también, análisis centrados en el microcontexto de la inserción, próximos a las explicaciones evolutivas de la carrera, que definen los problemas de ajuste en función de los requisitos laborales del individuo y de las demandas del empleador.

No obstante, en la actualidad, estamos en un momento de integración de planteamientos y de claro reconocimiento de la implicación mutua de factores académicos y laborales, macro y microcontextuales, de factores socioeconómicos y de otros factores ligados al propio sujeto agente del proceso de inserción. Esta integración queda justificada por varias razones: a) por la dificultad que entraña la generación de empleo desde la actuación sobre un único factor, b) por la constatación de que tanto el sistema educativo como el mercado de trabajo definen los condicionantes estructurales más importantes, c) porque esta complejidad invita a entender el concepto de transición como proceso que incluye varias fases o itinerarios en el acceso y permanencia en el mercado de trabajo y d) porque aunque el proceso de inserción tiene una resolución de carácter estrictamente individual, a nivel colectivo, se pueden tipificar parámetros comunes para un conjunto de sujetos que comparten situaciones y que han seguido trayectorias similares.

De entre los estudios que en nuestro contexto han tomado como punto de referencia el proceso de inserción laboral que recorren los graduados desde que terminan sus estudios hasta que obtienen un trabajo estable, se encuentran los de Casal, Masjuán y Planas (1991), García y Gutiérrez (1995), Peiró y García Montalvo (2001). En términos generales, se puede afirmar que estos estudios consideran la historia, académica y laboral, de los estudiantes como una buena base de información para analizar la relación entre el sistema educativo y la realidad laboral.

Casal, Masjuán y Planas (1991) en su estudio de los itinerarios de los jóvenes-adolescentes (hasta los 19 años) y de los jóvenes-adultos (19-25 años) barceloneses, plantean precisamente el significado de itinerario formativo y laboral fundamentado en la idea de transición como proceso continuado que incluye una diversidad de caminos en el que la clase social junto con el género y el componente territorial son factores básicos de carácter explicativo en los procesos de paso hacia la actividad. Abundando en el tema, Requena (1991) insiste en que las desigualdades adscritas al capital social por la procedencia fami- 
liar, afectan tanto al acceso a un puesto de trabajo como a la calidad alcanzada en el mismo, puesto que es precisamente el lugar ocupado por los padres en el entramado social, el que posibilita a su vez redes sociales informales que facilitan la información o las posibilidades de colocación (pág. 110).

De Miguel (1998) en el estudio de los itinerarios profesionales de los universitarios asturianos distingue entre componentes de carácter estructural externos al sujeto, que configuran las ofertas de empleo en un contexto (como son desequilibrios entre oferta y demanda, exigencia de formación especializada) y componentes de carácter individual (como nivel académico, información y orientación de la que se dispone, expectativas académico-profesionales), que contribuyen a la construcción de la imagen que cada sujeto tiene de sí mismo como individuo con posibilidades de organizar su vida con independencia del contexto familiar. Modelos como los de Montané (1990) y Blanch (1990), que describen el proceso de inserción desde la perspectiva del logro ocupacional, de igual modo contemplan la integración de factores sociales y personales, si bien se distinguen por la importancia que conceden a variables de tipo cognitivo-conductual.

De forma particular, coincidimos con Figuera (1996) acerca de que el estudio del proceso de inserción de la población universitaria exige analizar factores muy diversos situados en dos niveles de concreción distintos, pero explicativos de las posibilidades de inserción profesional. Uno, macroeconómico, que analiza la inserción en el marco del binomio universidad-mercado laboral y en términos de grado de ajuste (cuantitativo y cualitativo) entre la oferta de titulados y las necesidades y demandas del mercado laboral. Otro, microeconómico, representado por el proceso de convergencia entre la actividad exploratoria del universitario (búsqueda, implicación y actitud, grado de conocimiento y foco de la búsqueda) y el proceso de reclutamiento seguido por el empleador. Entre ambos contextos, y como un importante elemento modulador de la eficacia de las variables personales, la autora sitúa el título académico unido a la variable «tipo de institución que lo expide». En su opinión, el contexto inmediato de conducta de transición se sitúa precisamente en el enclave de las posibilidades del título en el mercado de trabajo cualificado y dentro de un contexto socio-geográfico determinado.

Conforme a todo ello, en la reconstrucción del itinerario profesional de los graduados objeto del presente estudio se ha tenido en cuenta dos niveles: uno, individual, otro, contextual o estructural. Se parte de la hipótesis de que los procesos de inserción en el mundo laboral deben interpretarse en función de los niveles formativos y características personales y, también, en función de las características del contexto de inserción y de las condiciones del mercado de trabajo que determinan, en un momento dado, el acceso a un campo profesional. La conjunción de estos dos niveles no sólo permite un análisis más comprehensivo de la trayectoria del sujeto, sino que además explica que, en el recorrido entre el mundo educativo y el laboral, puedan existir vías o soluciones variadas. Precisamente, estos patrones e itinerarios son los que pretende hacer emerger nuestro estudio, para lo cual se ha configurado un sistema de factores y variables susceptible de ser predictor de éxito de la inserción profesional de los titulados castellano-manchegos. Dicho sistema se representa en la Figura 1.

Como puede observarse, en la parte inferior izquierda se ha ubicado el macrocontexto cuya vertebración la conforman distintos elementos como son el marco político, el tejido social, etc. Esta configuración del macrocontexto determina en nuestra sociedad las vías de ac- 


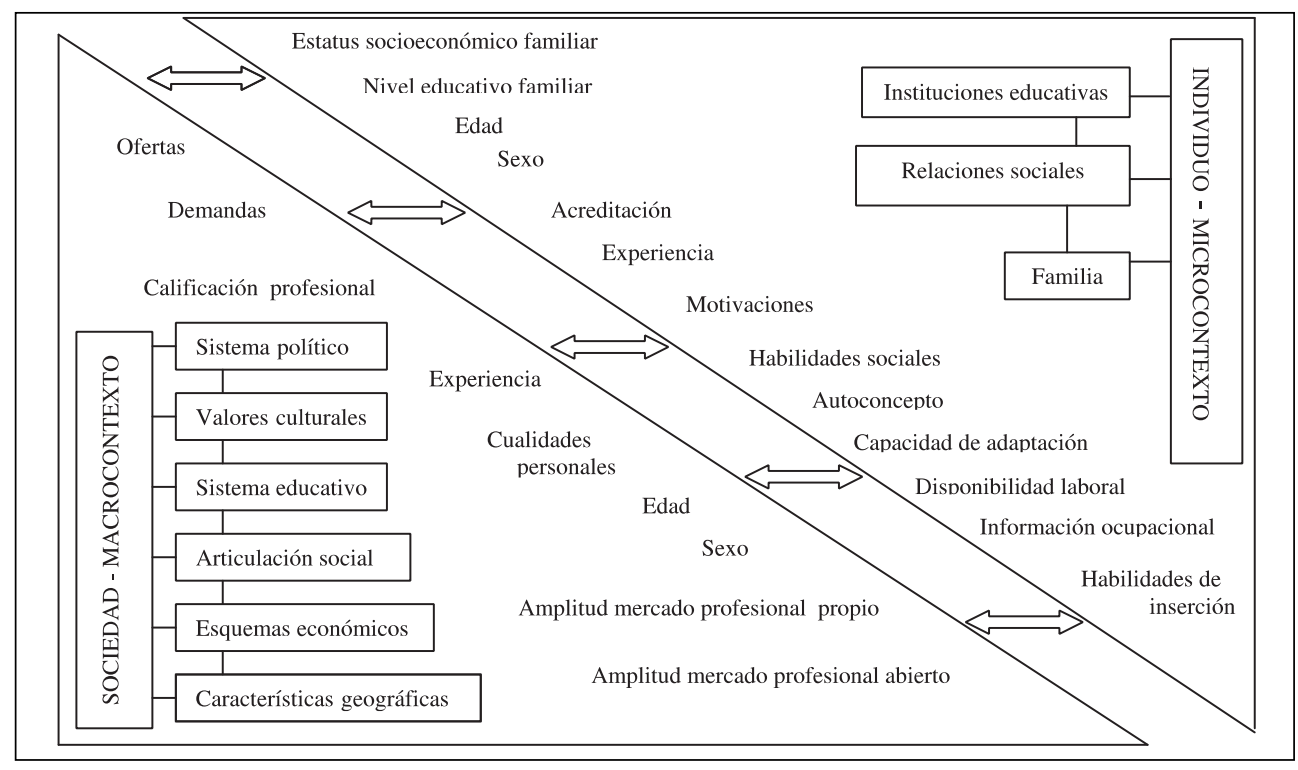

\section{FIGURA 1. \\ Factores y variables susceptibles de intervenir en el proceso de inserción profesional.}

ceso al ejercicio de la profesión cuyas variables se agruparían en distintos factores, el primero de ellos se refiere al mercado laboral que condiciona las posibilidades de obtener empleo (Montané, 1990); así, las oscilaciones entre la oferta y la demanda determinan la situación del mercado y la competencia entre los aspirantes. Otro factor importante son los requisitos que se demandan por parte de los empleadores y que estarían íntimamente ligados a las variables de titulación y a la institución que otorga las credenciales educativas, experiencia laboral en el sector, cualidades personales que pueden ir desde la capacidad de adaptación a las exigencias del mercado de trabajo hasta la habilidad para establecer relaciones sociales positivas, edad y sexo. Otras variables fundamentales son las que hacen referencia al prestigio y conocimiento que desde el macrocontexto se tiene acerca de la profesión y que se concretan, por un lado, en la amplitud del mercado laboral que le es propio a cada profesión y, por otro, en la amplitud del mercado profesional abierto en el cual es posible competir junto a otros profesionales.

Siguiendo con la Figura 1, y en la parte superior derecha, puede observarse la caracterización del microcontexto donde se ubica el individuo y que vendría determinada, en primer lugar, por la familia con su estatus socioeconómico, su nivel cultural y sus expectativas respecto a la educación y el trabajo y que distintos autores consideran variables básicas en la inserción (Consejo de Universidades, 1988; Fernández Enguita, 1990); en segundo lugar, el soporte social del que dispone el individuo $\mathrm{y}$, por último, las instituciones educativas tanto formales como no formales a las que acude.

De arriba a abajo se han dispuesto las variables supuestamente predictoras de la inserción profesional, así, las primeras hacen referencia a las variables de procedencia familiar 
que se acaban de mencionar. A continuación, la edad, el sexo, la acreditación educativa y la experiencia conformarían el currículum vitae del demandante; por otro lado, las motivaciones que sustentan la búsqueda de empleo, el autoconcepto y las habilidades sociales, tendrían que ver con las cualidades personales de cada individuo, a las que habría que sumar la disponibilidad laboral en lo que se refiere a la intensidad de la búsqueda de empleo y a las alternativas que se contemplan. Finalmente, la estrategia de búsqueda para afrontar la situación de inserción se concreta en habilidades como son la elaboración del currículum, el método de búsqueda de empleo y la información ocupacional de la que se dispone. Todo este conjunto de variables pretende manifestar, tal y como señalan los enfoques evolutivos en el ámbito de la orientación profesional, que la inserción se resuelve en un momento puntual, pero que se construye a lo largo de todo un proceso que se dilata en el tiempo o tiene consecuencias de distinto signo sobre el bienestar psicológico del sujeto, dependiendo del ciclo económico, en expansión o en recesión, en el que se busca empleo. Precisamente, en el marco de desarrollo de la carrera profesional, el acceso al empleo se produce por fases como ilustración de la evolución de la historia laboral del sujeto.

Para terminar, en la figura se ha querido resaltar la idea de interacción entre ambos contextos mediante las flechas bidireccionales. Con ello se representa la intención de detectar posibles situaciones de transición como resultado de la combinación entre ocupación, desempleo y formación.

\section{Objetivos del estudio y características de la muestra}

La investigación se interesa por la historia laboral previa del alumnado, pero sobre todo atiende a sus posibilidades de inserción profesional como psicopedagogos para detectar, precisamente, cuáles son las dificultades que se plantean en los primeros momentos de entrada al mercado laboral. El estatus de inserción se estudia pues en la etapa profesional inicial del alumnado de Psicopedagogía, siendo conscientes de que este hecho no puede reducirse a la consecución de este primer empleo ya que, precisamente, los itinerarios se inician con las primeras experiencias laborales y, una vez medianamente definidos, el problema que se plantea no es tanto de inserción como de estabilización en la actividad profesional (Feller y Walz, 1997).

Así pues, los resultados que aquí presentamos responden a la primera fase de nuestro trabajo de investigación cuyo objetivo es la identificación de los principales elementos que componen la trayectoria académica y laboral del alumnado de Psicopedagogía de la UCLM. Posteriores fases de este proyecto de carácter longitudinal tendrán como objetivo el seguimiento de estos alumnos y la evaluación del grado de inserción profesional logrado. A partir de ahí, contando con los datos que ahora presentamos y con el éxito en la inserción profesional de cada alumno, el análisis tendrá como propósito fundamental la identificación de los itinerarios que favorecen la inserción y de las posibles variables predictoras que estuvieran mediando en tal proceso.

Para la consecución del objetivo planteado evaluamos a una muestra de alumnos de las dos primeras promociones de la titulación de Psicopedagogía de la Facultad de Ciencias de la Educación y Humanidades. Así, la población estaría constituida por la totalidad de estos alumnos y si bien la convocatoria ser realizó para la población completa, la evaluación real se llevó a cabo con aquellos que voluntariamente decidieron colaborar en este estudio, estando la muestra final compuesta por un total de 114 alumnos cuyas características se detallan en la tabla 1. 


\section{Recogida de información: variables e instrumentos}

Las variables evaluadas se agrupan en las siguientes áreas:

A) Caracterización sociodemográfica: curso, edad, sexo, provincia de procedencia, tamaño del municipio de origen, número de hermanos, nivel de estudios de los hermanos y de los padres y renta familiar mensual.

B) Trayectoria académica: número de años empleados en cursar los estudios de Magisterio, la especialidad elegida, la nota media obtenida en la Diplomatura, motivaciones para estudiar Psicopedagogía, conocimiento de cada itinerario formativo, escolar y sociolaboral, antes de la matriculación y la intención de continuación de estudios de Postgrado.

C) Trayectoria laboral: experiencia laboral previa, estrategias de búsqueda utilizadas y la relación contractual establecida en la actualidad.

D) Estrategias y conductas ante la búsqueda de empleo: información que tienen los alumnos sobre organismos y empresas para solicitar empleo, el conocimiento acerca de elaboración del currículum vitae, las entrevistas de selección o pruebas psicotécnicas; el conocimiento de idiomas y las destrezas relativas a las Nuevas Tecnologías de la Información y las Comunicaciones.

E) Actitudes y competencias personales: Autoconcepto y Habilidades Sociales.

La recogida de información se ha realizado a través de los siguientes instrumentos:

- «Cuestionario para la Detección de los Itinerarios Formativos y de Inserción Laboral de los Licenciados de Psicopedagogía por la Universidad de Castilla-La Mancha». Este es un cuestionario de lápiz y papel, elaborado ad hoc por el equipo investigador para la evaluación de las cuatro primeras áreas. Las respuestas tomaron diversos formatos en función de la variable evaluada, habiéndose utilizado mayoritariamente respuestas de elección múltiple para variables nominales y escalas tipo Likert. La estructura del cuestionario responde a la presentación de las variables que se ha detallado en las líneas anteriores. Así, quedaba finalmente compuesto por cuatro áreas: a) caracterización sociodemográfica, b) trayectoria académica, c) trayectoria laboral, y d) estrategias y conductas ante la búsqueda de empleo; quedando cada una de las áreas compuesta por un total de 19, 13, 14 y 21 ítems, respectivamente, que tenían como objetivo obtener información detallada sobre las dimensiones presentadas en las líneas previas.

En este cuestionario muchas de las variables tienen carácter cualitativo, por lo que se ha superpuesto la validez de contenido a la obtención de índices estadísticos sobre fiabilidad o consistencia interna. Así, para la construcción del cuestionario se han tenido en cuenta tanto los instrumentos previamente utilizados en estudios de similares características (De Miguel, 1998; Palafox, 1997), como las teorías y conceptos a partir de los que se constituye, primando así la corrección teórica y metodológica sobre la estadística.

- Cuestionario AF-5, diseñado por García y Musitu (1999). Este cuestionario evalúa el autoconcepto de forma multidimensional, definiendo cinco factores diferentes: el físico, el social, el familiar, el académico/laboral y el emocional.

- Escala de Habilidades Sociales (EHS) de Gismero (2000). Este cuestionario, además de disponer de una puntuación global, permite obtener puntuaciones específicas relativas a distintas situaciones sociales: autoexpresión, defensa de los propios derechos, 
expresión de disconformidad, decir no y cortar interacciones, hacer peticiones e iniciar interacciones con el sexo opuesto.

\section{Discusión de resultados}

Con relación a las variables sociodemográficas, (tabla 1) tenemos que el 80,7\% de los estudiantes de Psicopedagogía son mujeres, que proceden en un $86 \%$ de Castilla-La Mancha y, en su mayoría, de la provincia de Cuenca (54,4\%), más concretamente, en un 30,3\% de los casos de núcleos de población iguales o menores a 10.000 habitantes. La movilidad del alumnado es elevada puesto que el $63,2 \%$ ha tenido que abandonar su lugar habitual de residencia para cursar estos estudios. A este respecto, cabe apuntar que el soporte familiar y la política de

TABLA 1: Estadísticos descriptivos de las variables sociodemográficas.

\begin{tabular}{|l|c|}
\hline & $\mathbf{N}(\mathbf{\%})$ \\
\hline Curso & \\
- Primero & $67(58,7)$ \\
- Segundo & $47(41,3)$ \\
\hline Edad & $24,7-5,12(21-44)^{*}$ \\
\hline Sexo & \\
- Varones & $22(19,3)$ \\
- Mujeres & $92(80,7)$ \\
\hline Provincia de procedencia & \\
- Cuenca & $62(54,4)$ \\
- Albacete & $15(13,2)$ \\
- Ciudad Real & $12(10,5)$ \\
- Guado & $8(7)$ \\
- Otras provincias de España & $1(0,9)$ \\
\hline Tamaño del municipio de origen & $16(14)$ \\
- Menos de 1.000 hab. & $10(9,2)$ \\
- Entre 1.001 y 5.000 hab. & $11(10,1)$ \\
- Entre 5.001 y 10.000 hab. 10.001 y 50.000 hab. & $12(11)$ \\
- Entre 50.001 y 100.000 hab. & $51(46,8)$ \\
- Más de 100.000 hab. & $6(5,5)$ \\
\hline Número de hermanos & $19(17,4)$ \\
- Ninguno & \\
- Uno & $5(4,4)$ \\
- Dos & $42(36,8)$ \\
- Cuatro & $32(28,1)$ \\
- Cinco & $22(19,3)$ \\
\hline
\end{tabular}

* Media - desviación típica (rango) 
TABLA 1 (Cont.): Estadísticos descriptivos de las variables sociodemográficas.

\begin{tabular}{|c|c|c|}
\hline & \multicolumn{2}{|c|}{ N (\%) } \\
\hline $\begin{array}{l}\text { Nivel de estudios de los hermanos } \\
\text { - Sin estudios o estudios primarios } \\
\text { - Al menos estudios secundarios } \\
\text { - Con estudios superiores }\end{array}$ & \multicolumn{2}{|c|}{$\begin{array}{c}16(14,7) \\
93(85,3) \\
41,3\end{array}$} \\
\hline $\begin{array}{l}\text { Estudios de los padres } \\
\text { - Sin estudios } \\
\text { - Primarios } \\
\text { - Bachiller elemental } \\
\text { - Bachiller superior } \\
\text { - Diplomado } \\
\text { - Licenciado } \\
\text { - Postgrado }\end{array}$ & $\begin{array}{c}\text { Padre } \\
12(10,6) \\
70(61,9) \\
10(8,8) \\
8(7,1) \\
8(7,1) \\
4(3,5) \\
1(0,9)\end{array}$ & $\begin{array}{c}\text { Madre } \\
19(16,8) \\
72(63,7) \\
6(5,3) \\
8(7,1) \\
7(6,2) \\
0(0) \\
1(0,9)\end{array}$ \\
\hline $\begin{array}{l}\text { Nivel de renta mensual familiar } \\
\text { - Menos de } 100.000 \text { ptas. } \\
\text { - Entre } 100.000 \text { y } 200.000 \text { ptas. } \\
\text { - Entre } 200.000 \text { y } 400.000 \text { ptas. } \\
\text { - Más de } 400.000 \text { ptas. } \\
\text { - NS/NC }\end{array}$ & \multicolumn{2}{|c|}{$\begin{aligned} & (3,5) \\
30 & (26,3) \\
26 & (22,8) \\
12 & (10,5) \\
42 & (36,8)\end{aligned}$} \\
\hline
\end{tabular}

becas y ayudas al estudio en esta región favorecen la estancia prolongada del alumnado fuera de sus lugares de origen para cursar estudios universitarios. No obstante, del hecho de que la titulación se nutra fundamentalmente de castellano-manchegos no necesariamente se desprende que todos vayan a terminar trabajando como psicopedagogos en la región.

En cuanto al nivel de estudios de los padres, observamos que más del $80 \%$ de ambos progenitores tienen estudios de bachiller elemental o inferior. Este dato, junto con la renta familiar, nos permite una aproximación superficial a la composición social del colectivo, así como hacer atribuciones sobre el posible capital relacional de nuestros titulados y su incidencia sobre la trayectoria laboral.

Los estadísticos descriptivos de las variables relativas a la trayectoria académica se presentan en la tabla 2. El mayor porcentaje de alumnos se agrupa en torno a la especialidad en Magisterio de Educación Infantil (30,4\%). El 88,6\% de los alumnos informa haber obtenido al menos un notable de nota media en la diplomatura de Magisterio, siendo ésta la nota de corte establecida para el acceso a la licenciatura. Las motivaciones de la mayoría de los alumnos $(84,2 \%)$ para estudiar Psicopedagogía son principalmente intrínsecas, como mejorar su formación en materia educativa, elevar su formación y nivel cultural, o conseguir cambios sociales a través de la educación; únicamente un 3,5\% informa de motivaciones fundamentalmente extrínsecas como tener mayor posibilidad de movilidad geográfica, elevar el nivel de ingresos u obtener mayor reconocimiento social. En cuanto al conocimiento de los estudios de Psicopedagogía que el alumnado tenía antes de matricularse, destaca que el 64,6\% no tenía información al respecto. Por último, merece la pena aportar el dato relativo a la intención de continuar estudios de postgrado, ya que la práctica totalidad de los alumnos $(99,1 \%)$ informó de tener intención de seguir estudiando después de finalizar la licenciatura, lo que pone de manifiesto un alto nivel de expectativas académicas. 
TABLA 2: Estadísticos descriptivos de la trayectoria educativa.

\begin{tabular}{|l|c|}
\hline & N (\%) \\
\hline Años empleados en cursar magisterio & \\
- Tres & $103(92)$ \\
- Cuatro & $6(5,4)$ \\
- Cinco & $3(2,7)$ \\
\hline Especialidad elegida & \\
- Infantil & $34(30,4)$ \\
- Primaria & $14(12,5)$ \\
- Audición y Lenguaje & $17(15,2)$ \\
- Educación Especial & $15(13,4)$ \\
- Educación Física & $3(2,7)$ \\
- Lengua extranjera & $19(17)$ \\
- Otros & $4(3,6)$ \\
\hline Nota media obtenida en magisterio & $6(5,4)$ \\
- Aprobado & \\
- Notable & $13(11,4)$ \\
- Sobresaliente & $97(85,1)$ \\
\hline Motivaciones fundamentales para estudiar Psicopedagogía & $4(3,5)$ \\
- Principal o únicamente intrínsecas & \\
- Principal o únicamente extrínsecas & $96(84,2)$ \\
- Ambas con peso similar & $4(3,5)$ \\
\hline Conocimiento previo de los itinerarios & $14(12,3)$ \\
- Sí & \\
- No & $40(35,4)$ \\
\hline Intención de realizar estudios de postgrado & $73(64,6)$ \\
- Sí & $12(0,9)$ \\
- No & \\
\hline
\end{tabular}

La exploración realizada también permite observar que estamos ante una muestra con buenos resultados académicos, pero que se matricula en Psicopedagogía sin tener toda la información que le permite tomar esta decisión en las mejores condiciones posibles. Sin embargo, al finalizar el primer curso, la tendencia se invierte y la información respecto a los itinerarios formativos (escolar y sociolaboral) es valorada en términos positivos. Además, es importante señalar que el alumno elige uno u otro itinerario en función de las posibilidades de trabajar que atribuye a una u otra especialización. En el análisis por grupos, pueden encontrarse explicaciones complementarias a este patrón de respuesta que demuestra la satisfacción respecto de la formación alcanzada. Solo el 19,7\% de los alumnos de primero considera que su actual nivel de formación no le va a permitir encontrar trabajo como psicopedagogo, frente al $27,5 \%$ de segundo curso. Estamos ante porcentajes no muy elevados pero que pueden esconder una percepción pesimista del mercado laboral, de desajuste entre la formación recibida y las demandas laborales o de carencia de determinados contenidos en el Plan de Estudios. En principio, y a la espera de un análisis más detallado del contexto de inser- 
ción, cabe apuntar que la formación conseguida por el alumnado de Psicopedagogía está vinculada a las necesidades educativas dominantes en la región y que este grado de adecuación, desde la perspectiva del alumno, puede verse reforzado mediante cursos de formación continua específica para la titulación de Psicopedagogía. La importancia de la formación complementaria en este terreno parece haber calado también en esta población, explicable por la necesidad de competir y el hecho de tener que hacerlo en las mejores condiciones.

Con relación a los resultados relativos al análisis de la trayectoria laboral, presentados en la tabla 3, diremos que el $61,9 \%$ de los alumnos ha trabajado en alguna ocasión durante los últimos tres años. En este período, el $97 \%$ de la muestra señala haber tenido entre 1 y 4 trabajos distintos con una duración inferior al año en el 54,6\% de los casos. Este primer dato nos describe un colectivo que realiza los tránsitos característicos de los años 80 y 90 entre períodos de formación, desarrollo de empleos inestables y situaciones de desempleo. Puesto que el colectivo no se dedica a la formación de manera exclusiva, en principio, es posible pensar que frente a quienes sí lo hacen o desarrollan ciclos formativos más prolongados, dispone de más tiempo para incorporarse al mundo laboral y cuando lo hace es con más experiencia.

Sobre las actividades desempeñadas en los últimos tres años, hay que señalar que el $71,4 \%$ afirma haber realizado trabajos relacionados con la educación en el marco de la transición magisterio-primer empleo. De ellos, el 30,8\% conforme a una contratación indefini-

TABLA 3: Estadísticos descriptivos de la trayectoria laboral.

\begin{tabular}{|l|c|}
\hline & N (\%) \\
\hline Trabajado en los últimos 3 años & \\
- Sí & $70(61,9)$ \\
- No & $43(38,1)$ \\
\hline Estrategias de búsqueda utilizadas* & \\
- Inscripción en el INEM & $42(71,2)$ \\
- ETT, agencia de colocación & $14(23,7)$ \\
- Cartas/CV a empresas & $33(55,9)$ \\
- Autoempleo & $0(0)$ \\
- Oposiciones & $29(49,2)$ \\
- Prensa y media & $23(39)$ \\
- A través de amigos y familiares & $7(11,9)$ \\
- Asociaciones y colegios profesionales & $6(10,2)$ \\
- Autoanuncio & $14(23,7)$ \\
\hline Contrato establecido & $5(8,5)$ \\
- Indefinido & \\
- Temporal & $14(41,2)$ \\
- De formación, prácticas o aprendizaje & $6(17,6)$ \\
- Estacional o de temporada & $0(0)$ \\
- Sustitución & $3(8,8)$ \\
- Autónomo & $3(8,8)$ \\
- Beca & $1(2,9)$ \\
- Otros & $5(14,7)$ \\
\hline
\end{tabular}

* Categorías no excluyentes 
da como maestros en activo, y el 35,2\% de forma temporal, estacional o por sustitución. Entre los alumnos de primer curso, el porcentaje que ha trabajado en los últimos tres años, lo ha hecho sin contrato o con contratos temporales y en trabajos relacionados con la educación que tienen un gran efecto sobre la incipiente socialización profesional (voluntariado, clases particulares, como becarios). En el momento de la exploración, el 27,3\% desempeñaba un trabajo relacionado con la educación - para el que, en opinión del $90 \%$ estaba sobrecualificado-, frente al 6,4\% cuyo trabajo no guardaba ninguna relación con lo educativo. El $36,4 \%$ estaba en situación de inactividad.

A la vista de estos resultados, puede apuntarse que la trayectoria laboral explorada describe un cierto desempleo en alternancia con el empleo inestable y compaginándolo con la continuación de estudios. Desde este punto de vista cabe afirmar que el alumnado de Psicopedagogía ya ha realizado una primera transición con las características propias que supone el acceso al ejercicio profesional del magisterio y que las situaciones de inserción definidas por el estatus de empleo y las condiciones descritas, en cierto sentido, le habilitan para una segunda transición.

Los datos relativos a las variables referidas a las estrategias y actitudes ante la búsqueda de empleo son presentados en la tabla 4. Las variables en este punto evaluadas, junto con las relativas a las actitudes y competencias personales, adquieren una importancia notable en el estudio. Frente a las condiciones del mercado de trabajo y de cambio en las condiciones de inserción, las estrategias de búsqueda y actitudes personales que cada sujeto posee aparecen como formas diferenciadas de enfrentarse a esa situación objetiva. Digamos que las reglas de juego son iguales para todos, pero cada individuo se enfrenta a la inserción como a cualquier otro proceso de interacción, con sus características previas, su capital cultural, social y relacional, expectativas y comportamiento a la hora de su acceso a la vida laboral. Y, también, haciendo uso de unas u otras estrategias de búsqueda activa.

Sobre este tema, llama la atención que el $69,4 \%$ de los alumnos no tiene información acerca de organismos y empresas para solicitar empleo como psicopedagogo y además no sabría cómo conseguirla. La información respecto a las salidas profesionales, así como las redes existentes que facilitan el contacto y la colocación aparece como deficitaria. El colectivo analizado no se caracteriza por una gran diversificación de las estrategias de búsqueda de empleo;

TABLA 4: Estadísticos descriptivos de las variables relativas a estrategias ante la búsqueda de empleo.

\begin{tabular}{|l|l|}
\hline & N (\%) \\
\hline Información sobre organismos/empresas para solicitar empleo & \\
- Tiene información & $20(18,5)$ \\
- No tiene información pero sabría conseguirla & $13(12,03)$ \\
- No tiene información y no sabe cómo conseguirla & $88(69,4)$ \\
\hline Información de técnicas de búsqueda de empleo* & \\
- Cartas de presentación & $60(56,6)$ \\
- Currículum Vitae & $92(83,6)$ \\
- Pruebas psicotécnicas & $18(17)$ \\
- Entrevista de selección & $27(25,5)$ \\
- Seguimiento de solicitudes & $18(17,1)$ \\
\hline
\end{tabular}

* Porcentaje de respuestas afirmativas. 
no obstante, las técnicas específicas más conocidas son la preparación del currículum vitae y de cartas de presentación, mientras que se tiene poca información acerca de la realización de pruebas psicotécnicas, entrevistas de selección y seguimiento de solicitudes de empleo.

En lo que a resultados referidos al conocimiento de idiomas y nuevas tecnologías como competencias que favorecen la empleabilidad, se observan carencias a tener en cuenta. Respecto a las actitudes y competencias personales (autoconcepto y habilidades sociales), por el contrario, se registran resultados óptimos que bien pudieran estar apuntando hacia efectos de deseabilidad social, lo que deviene un dato significativo susceptible de seguimiento. Las medias obtenidas en cada una de las dimensiones de los cuestionarios de autoconcepto y de habilidades sociales se detallan en los gráficos 1 y 2 respectivamente

Para terminar y, a la luz de los resultados obtenidos, quisiéramos poner de manifiesto algunas conclusiones e hipótesis de trabajo que orientan fases ulteriores de este proyecto. En primer lugar, es importante señalar que los datos recogidos ratifican la modificación general que se ha producido en la estructura de la fuerza de trabajo castellano-manchega en términos de cualificaciones, con una clara incidencia en la definición del mercado de trabajo. Conforme a la territorialidad de la oferta y demanda de psicopedagogos, cabe señalar que la movilidad en tanto que característica del colectivo descrito puede interpretarse como tendencia que favorecerá su redistribución por toda la Junta de Comunidades. No obstante, aparece como dificultad que en la región no existe una clara estructura ocupacional para los psicopedagogos: limitada en el ámbito formal, por explotar en el no formal. En principio, el mercado laboral de los psicopedagogos castellano-manchegos es tan difuso como en el resto del territorio. Que el titulado carezca de información acerca de los organismos y empresas en los que puede solicitar empleo como psicopedagogo, se explica por la propia indefinición de los campos de actuación en los que tiene cabida puesto que, si bien el perfil del psicopedagogo se fundamenta en las necesidades psicoeducativas de la sociedad, no se ha llegado a una clara concreción de las funciones y perfiles en las ofertas de empleo dirigidas a estos titulados que permita una relación fluida entre oferta y demanda.

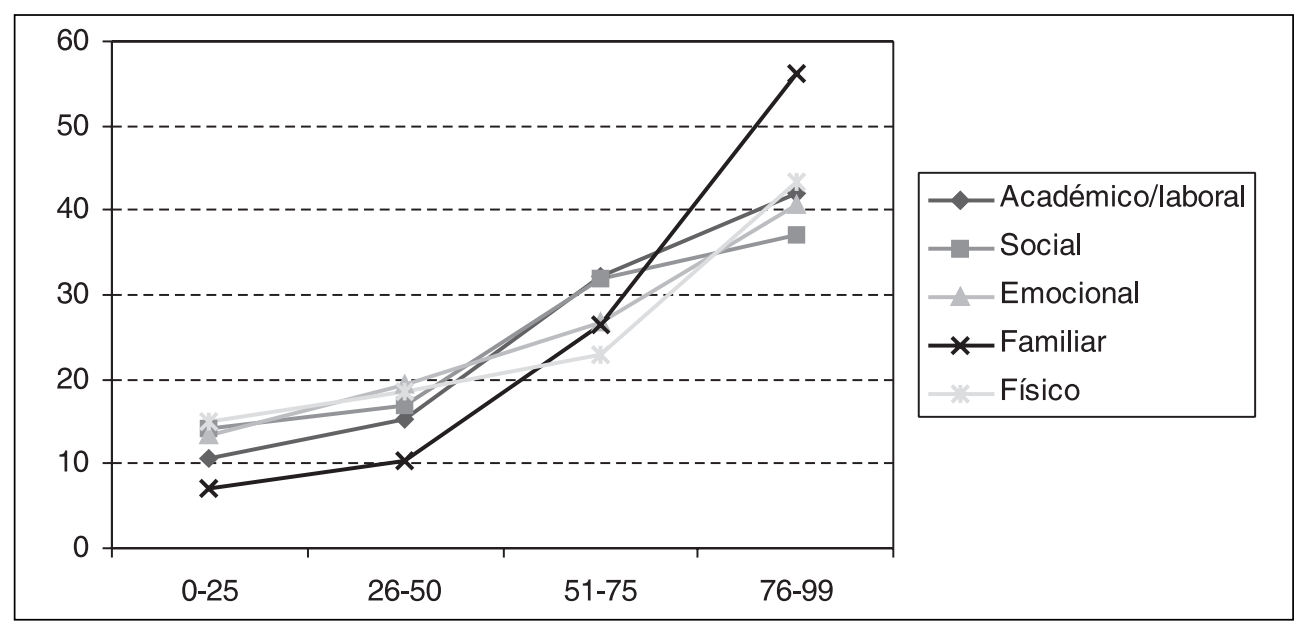

GRÁFICO 1.

Resultados obtenidos en las distintas dimensiones de la Escala AF5 (Autoconcepto). 


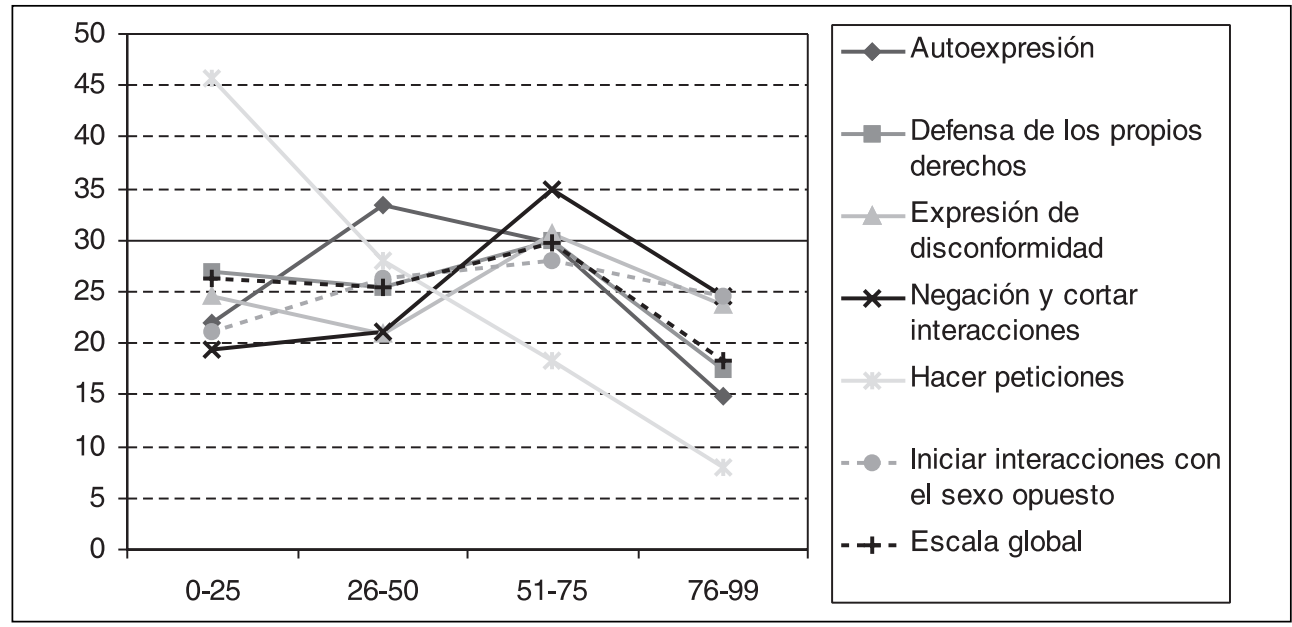

\section{GRÁFICO 2. \\ Resultados obtenidos en las distintas dimensiones de la Escala EHS (Habilidades Sociales).}

Con respecto a la inserción profesional de este colectivo, aunque de momento no se dispone de datos concluyentes, sí que se está en condiciones de afirmar que la carencia de estrategias de desarrollo de la carrera y la no utilización de un método de búsqueda de empleo funcionarán como barreras a la inserción. Junto a estos factores, uno tercero que el estudio ha detectado como importante es el que se refiere al grado de identificación que el alumnado ha conseguido con el rol de psicopedagogo puesto que se considera como variable que sostiene en el tiempo la búsqueda activa de empleo como tal. De entre los indicadores que el estudio está utilizando para comprobar este comportamiento, se encuentra el seguimiento de los alumnos ya evaluados que han participado en el proceso de oposiciones recientemente celebrado en nuestra región para el acceso al cuerpo de maestros de primaria, frente a los que están valorando otras opciones (autoempleo, acceso al cuerpo docente de secundaria, psicopedagogo en instituciones variadas).

Por último, señalar que estamos ante una ocupación que sufrirá una expansión moderada, por lo que la movilidad regional y la mayor acumulación de capital formativo adecuado a las necesidades psicopedagógicas del contexto actuarán como factores de potenciación del proceso de inserción profesional del colectivo estudiado. En este sentido es importante señalar que la absorción de esta fuerza de trabajo por parte del mercado es lo que precisamente centra nuestros esfuerzos ${ }^{1}$. De momento simplemente se puede avanzar que la gestión y organización del empleo para los psicopedagogos en esta región pasa por un mayor conocimiento por parte de los empleadores de las funciones y perfil de estos profesionales.

1. Para el estudio del contexto de inserción se están desarrollando distintas estrategias. Entre ellas, destacamos las entrevistas en profundidad con los tutores de Prácticum de la red de centros colaboradores con la Facultad y el análisis de las ofertas de empleo que se publican en la región. La triangulación de los datos así obtenidos nos va a permitir dibujar con mayor grado de certeza el mapa de opciones que se presentan a nuestros titulados. 


\section{Referencias bibliográficas}

Blanch, J. M. (1990). Del viejo al nuevo paro. Un análisis psicológico y social. Barcelona: PPU.

Casal, J., Masjuán, J. M. y Planas, J. (1991). La inserción social y profesional de los jóvenes. Barcelona: ICE-CIDE.

Consejería de Educación. (1999). Libro Blanco de la Educación en Castilla-La Mancha. Junta de Comunidades de Castilla-La Mancha.

Consejo de Universidades (1988). La situación y perfil del desempleo y subempleo de los titulados universitarios. Madrid: MEC.

De Miguel Diaz. M. (1998). Los estudios universitarios y la inserción en el mundo profesional. Seguimiento de una cohorte. Servicio de Publicaciones: Universidad de Oviedo.

Feller, R., y Walz, G. (Eds.) (1997). Career transitions in turbulent times. Exploring work, learning and careers. University of North of Carolina: ERC/CASS Publications.

Fernández Enguita, M. (1990). Educación, formación y empleo en el umbral de los noventa. Madrid: MEC.

Figuera Gazo, P. (1996). La inserción del universitario en el mercado de trabajo. Barcelona: EUB.

García, J. y Gutiérrez, R. (1995). Inserción laboral de los jóvenes. Revista asturiana de Economía, 2, $55-80$.

García, F y Musitu, G. (1999). Autoconcepto forma 5 (AF-5). Madrid: TEA Ediciones.

García Montalvo, J. Palafox, J, Peiró, J. M. y Prieto, F. (1997). La inserción laboral de los jóvenes de la Comunidad Valenciana. Valencia: Fundación Bancaja.

Gismero González, E. (2000). Escala de Habilidades sociales (EHS). Madrid. TEA Ediciones.

Masjuan, J. M., Zaldívar, M. y Vivas, J. (1992). Estudi des itineraris d'ocupació i fomració dels nous titulats universitari. Barcelona: ICE de la Universitat Autónoma de Barcelona i Departament d'Ensenyament de la Generalitat de Catalunya.

Montané, J. (1990). Transición de la escuela al trabajo: enfoques y técnicas. En actas de las VI Jornadas Nacionales de Orientación Escolar y Profesional. Canarias 29 octubre-3 noviembre de 1990.

Palafox, J. y Pérez, F. (1997). Capital humano. Educación y empleo en la comunidad valenciana. Valencia: Fundación Bancaja.

Peiró, J. M. y García Montalvo, J. (2001). Capital humano. El mercado laboral de los jóvenes: formación, transición y empleo. Valencia: Fundación Bancaja.

Requena, F. (1991). Redes sociales y mercado de trabajo: elementos para una teoría del capital relacional. Madrid: Siglo XXI. Centro de Investigaciones Sociológicas.

Roquero, E. (1995). La inserción a la vida activa. Granada: Servicio de Publicaciones de la Universidad de Granada.

Spenner, K. (1985). The upgrading and downgrading of occupation. Review of Educational Research, 55 (2), 125-154.

Fecha de recepción: 10-IX-01

Fecha de revisión: 15-III-02

Fecha de aceptación: 5-XI-02 